Mal J Nutr 27(1): 027-040, 2021

\title{
REVIEW
}

\section{Malnutrition and its risk factors among children and adolescents with intellectual disability (ID) in Asian countries: A scoping review}

\author{
Siti Fathiah Mohamed, Soo Kah Leng* \& Divya Vanoh
}

Nutrition and Dietetics Programme, School of Health Sciences, Universiti Sains Malaysia, Kubang Kerian, Kelantan, Malaysia

\begin{abstract}
Introduction: Children with intellectual disability (ID) have higher probability of experiencing poor health status due to their limited ability to understand and assess information about nutrition and health. Malnutrition remains a significant health problem as it is prevalent among both typically developing and disabled children. This scoping review aims to discuss the prevalence of malnutrition among children and adolescents with ID in Asian countries. Methods: The review was based on the PRISMA-ScR method. A systematic electronic search was conducted using databases namely PUBMED, Science Direct, Scopus, and Google Scholar for articles published from 2006-2019, written in English and involved studies in Asian countries. Results: A total of 255,100 article's titles were identified and only 17 articles that met the inclusion criteria were included for further analysis. The findings revealed that the range of prevalence for underweight was 5.7\%-76.3\% and $28.9 \%-45.0 \%$ for stunting. The prevalence estimated for overweight was $3.9 \%-46.6 \%$, while obesity was $1.6 \%$ $26.6 \%$. The body mass index values ranged from $17.4 \pm 4.3 \mathrm{~kg} / \mathrm{m}^{2}-23.0 \pm 6.3 \mathrm{~kg} / \mathrm{m}^{2}$. There were several risk factors related to malnutrition such as co-morbidity factors, behavioural factors, and parental factors. Conclusion: This review demonstrated concern regarding the prevalence of malnutrition among children and adolescents with ID, which is high in certain Asian countries. It was varied and thus difficult to compare due to different reference standards and definitions used for malnutrition. Establishing nutrition and health-related intervention programmes can help to prevent further rise in the prevalence of malnutrition in this group.
\end{abstract}

Keyword: Intellectual disability, malnutrition, overweight, obesity, underweight

\section{INTRODUCTION}

Intellectual disability (ID) is referred as a condition characterised by impaired intelligence and social functioning which begins before adulthood and affects normal development (WHO, 2020). Based on the American Association on Intellectual and Developmental Disabilities (AAIDD), intellectual disability, which was previously known as mental retardation, is defined as "the presence of three coexisting features, namely significant sub-average intellectual functioning, deficits or

\footnotetext{
*Corresponding author: Dr Soo Kah Leng

Nutrition and Dietetics Programme, School of Health Sciences, Universiti Sains Malaysia,

Kubang Kerian, Kelantan, Malaysia

Tel: (6) 097677631; Fax: (6)097677515; E-mail: sook1@usm.my

doi: https://doi.org/10.31246/mjn-2020-0067
} 
impairments in adaptive skills, and onset before 18 years of age" (Shea, 2012). The United Nations Children's Fund (UNICEF) (2018) estimated that there are at least 93 million children with disabilities in the world, and this statistic could be higher due to unreported data. UNICEF further stated that 1 in 20 children aged 14 years or younger lives with moderate to severe disabilities. However, the data included all types of disabilities, not differentiated either by intellectual, physical or other disabilities. A meta-analysis found that the prevalence of ID among children and adolescents was $1.83 \%$ and the femaleto-male ratio varied between 0.4 and 1.0 (Maulik et al., 2011). Recently, McKenzie et al. (2016), who extended Maulik et al. (2011)'s work, reported that the prevalence of ID for all age groups was estimated to be $0.05 \%-1.55 \%$.

Some ID children would have comorbid conditions such as autism, attention deficit disorder (ADHD), Down syndrome, epilepsy, and anxiety (Eisenhower, Baker, \& Blacher, 2005; Jauhari et al., 2012; Krause et al., 2016). They have a higher probability of experiencing poor health status as compared to those without ID due to their limited ability to comprehend and assess information about nutrition and health. Hence, poor health status would expose them to the risk of getting communicable or non-communicable diseases as a secondary health condition (Bellamy, 2016). Nutritional status is an important indicator to determine the overall health status and wellbeing of children and adolescents with ID as these groups are vulnerable to suffer from malnutrition (under or over-nutrition).

The World Health Organization (WHO) (2020) defines malnutrition as "deficiencies, excesses, or imbalances in a person's intake of energy and/or nutrients; referring to three groups of conditions which are undernutrition, macronutrient-related malnutrition, and overweight/obesity". Malnutrition in this review will focus on undernutrition (wasting, stunting, and underweight) and overnutrition (overweight and obesity). The Global Nutritional Report (2020) reported a declining prevalence in underweight from $39.5 \%$ in the year 2000 to $33.7 \%$ in the year 2016 among children and adolescents (aged 5-19 years) in Asian countries. Meanwhile, overweight and obesity among children and adolescents showed a rising trend from 2000-2016, with a prevalence of $7.0 \%-17.3 \%$ and $1.7 \%-6.5 \%$, respectively. Even though the prevalence of underweight has decreased, it remains a problem because the decline is too slow, while overweight and obesity is rising rapidly.

Undernutrition among children and adolescents with ID may result due to a lack in nutritious food intakes following their dependency on feeding and food preparation. This situation might worsen when they lose weight due to other risk factors such as multiple health conditions, frequent infections, as well as limited access to quality health care. A systematic review reported that disabled children are three times more likely to be underweight and twice as likely to have wasting and stunting as compared to normal children (Humenixon \& Kuper, 2018). On the other hand, several studies found that children with disabilities had higher prevalence of overweight and obesity compared to children without disability (Bandini et al., 2015; Ogwu, 2012). A systematic review on overnutrition revealed that adolescents with ID have more risk of having overweight-obesity (1.54 times) and obesity (1.80 times) than typically developing adolescents (Maïano et al., 2016). The possible risk factors contributing to the rising level of overweight and obesity among children and adolescents with ID are age, gender, 
sedentary behaviour, higher intakes of energy-dense foods, co-morbidities, and genetic disorders (Krause et al., 2016; Segal et al., 2016). Disabled children who are obese will be vulnerable to remain obese during adulthood and are prone to get other health problems such as diabetes, hypertension, and cardiovascular disease (Raghi et al., 2016; Rimmer et al., 2010).

Thus, these evidences suggest that malnutrition remains one of the significant health problems among children and adolescents as it is not only prevalent among typically developing children, but also among disabled children. To our knowledge, no published review article has explored the existence of malnutrition among ID children and adolescents in Asian countries. Understanding the current situation and trends on malnutrition among Asian children and adolescents with ID will provide useful information to assist researchers and health professionals in identifying priority health programmes for the prevention and care of this vulnerable group. This scoping review aims to review the prevalence of malnutrition among children and adolescents with ID in Asian countries.

\section{MATERIALS AND METHODS}

\section{Sources of information and search strategy}

The present study is a scoping review that was designed based on the "Preferred Reporting Items for Systematic reviews and Meta-Analyses extension for Scoping Reviews (PRISMA-ScR)" method (Tricco et al., 2018). A systematic electronic search was conducted using four databases, namely PUBMED, Science Direct, Scopus, and Google Scholar to source for research papers published from 2006-2019 that were written in English. In addition, a hand search was carried out using the reference lists of relevant articles and previous literature reviews on related topics. The keywords that were used in the database machines comprised of malnutrition, nutritional status, underweight, obesity, overweight, body weight status, body mass index, children, adolescent, intellectual disability, and mental retardation. The search strategies included all possible combinations of key search terms which were: (a) (malnutrition) AND children OR adolescent AND (intellectual disability OR mental retardation), (b) (overweight OR obesity OR underweight) AND children OR adolescent AND (intellectual disability OR mental retardation) (c) body weight status AND children OR adolescent AND (intellectual disability OR mental retardation) and (d) body mass index AND children OR adolescent AND (intellectual disability OR mental retardation).

\section{Inclusion criteria}

Research articles that met these specific inclusion criteria were considered eligible for this review. Firstly, the study participants were presented with ID. Studies based on mixed samples of participants presented with multiple disabilities were also considered eligible if specific data regarding the prevalence of underweight, overweight, or obesity were available for children and/or adolescents with ID. Secondly, the age of the study participants had to be 19 years old and below, which consisted of children and/or adolescents. Studies on a single sample (children or adolescents) or mixed samples (children and adolescents) were considered eligible if specific data on malnutrition were available. Thirdly, this review only included original research articles of a cross-sectional study or survey that were written by the researchers who conducted the study. Lastly, only 
studies within 48 countries in the Asian continent were included.

\section{Study selection and data charting}

The articles were selected based on the PRISMA-ScR method (Tricco et al., 2018). The study selection was based on the objectives of the study which focused on malnutrition of children and adolescents with ID in Asian countries. The researchers eliminated irrelevant articles based on the inclusion and exclusion criteria, as well as those that did not answer the research questions. The researchers screened the eligibility of relevant articles separately, based on the titles and retrieved relevant abstracts. Abstracts that did not meet the scope of the study were excluded. Based on the eligible abstracts, copies of the full articles were retrieved from the databases by downloading the soft copies. The researchers checked and revised the full articles to determine whether they appeared to answer the research questions of the study to select the final full articles for review. The results of the article search were managed using the Mendeley software and the extracted data from the full articles were documented in Microsoft Word. Data charting was done independently by a researcher and verified by the other two researchers. General and specific information from the selected studies were extracted based on the inclusion criteria and charted in a table which included author(s), years of publication, country, sample characteristics (sample size, age, ID and co-morbidities), anthropometric measures / indices, reference standard/ classification criteria, and findings that were relevant to the objectives of the review.

\section{Collating, summarising, and reporting the results}

The characteristics and findings of the selected articles are presented in Table 1.
The results of the extracted data on the prevalence of malnutrition were analysed using descriptive statistics (percentage) to provide summary characteristics according to the scopes of malnutrition research. Several limitations of the studies were observed to determine the research gap and usefulness for future research in malnutrition.

\section{RESULTS}

Based on Figure 1, a total of 255,100 titles were identified through database searching. Next, 94 abstracts were included for the initial screening process, after which duplicates were removed and 40 were excluded due to several reasons (i.e., population, age group, and study design). The duplicated articles were managed by recording, keeping track, sorting the list, and checking them using the Mendeley software. Then, 34 full-text articles were assessed for eligibility; 13 articles were excluded due to non-Asian countries and four because they were articles that were reanalysed using data from the same study. Finally, only 17 articles met the inclusion criteria and were included in the review for analysis (Table 1). This

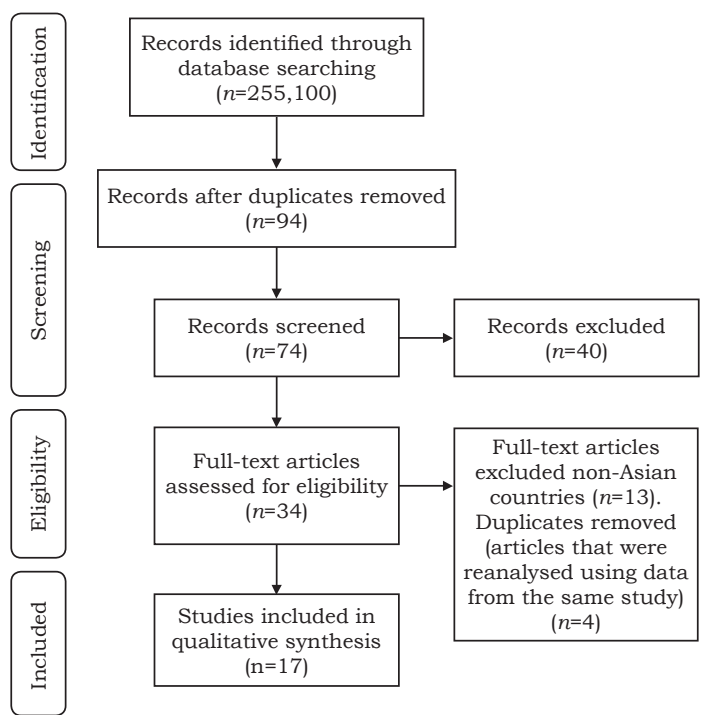

Figure 1. Flow chart of PRISMA-ScR method 
Malnutrition among children \& adolescents with ID in Asia

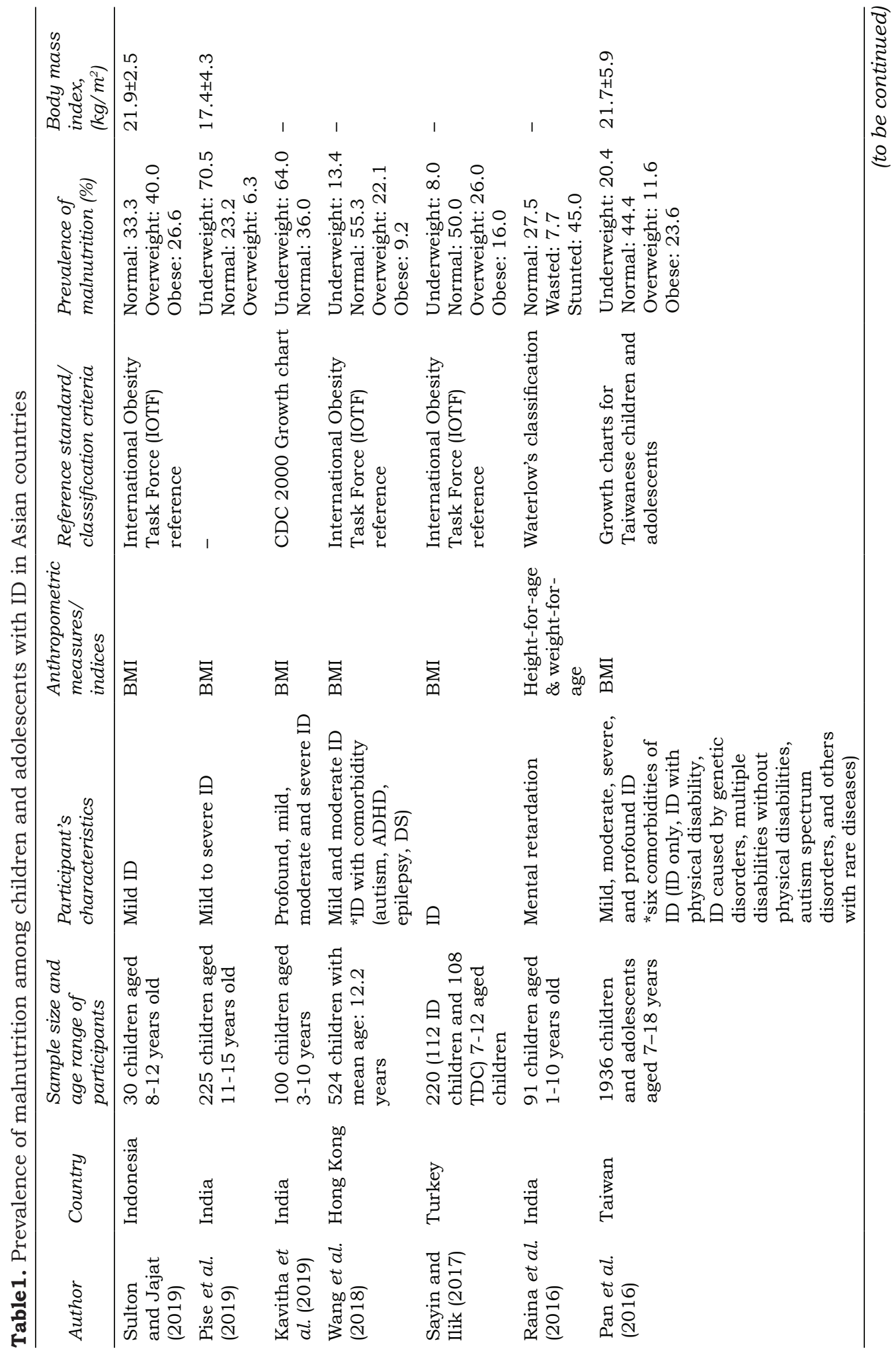




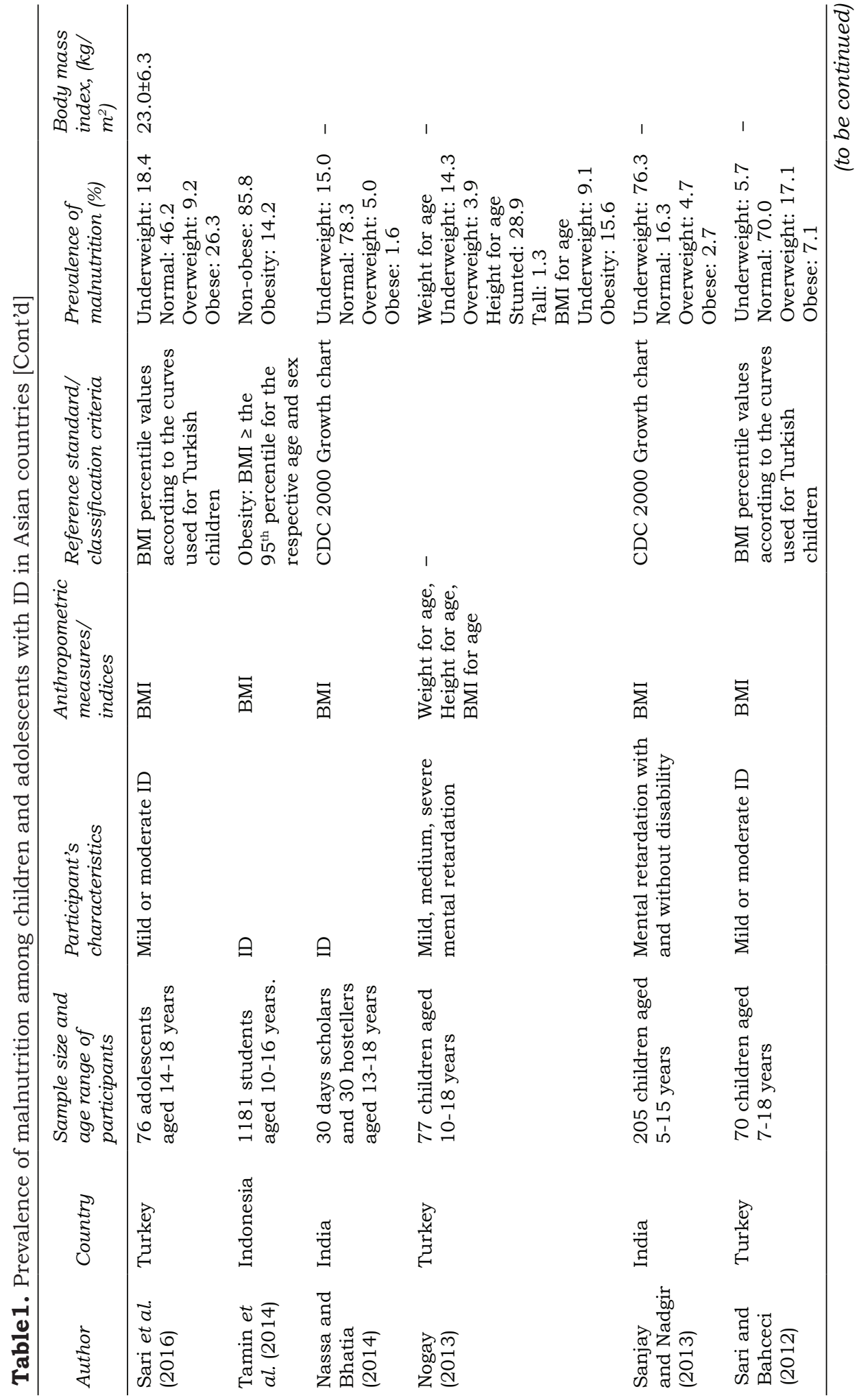




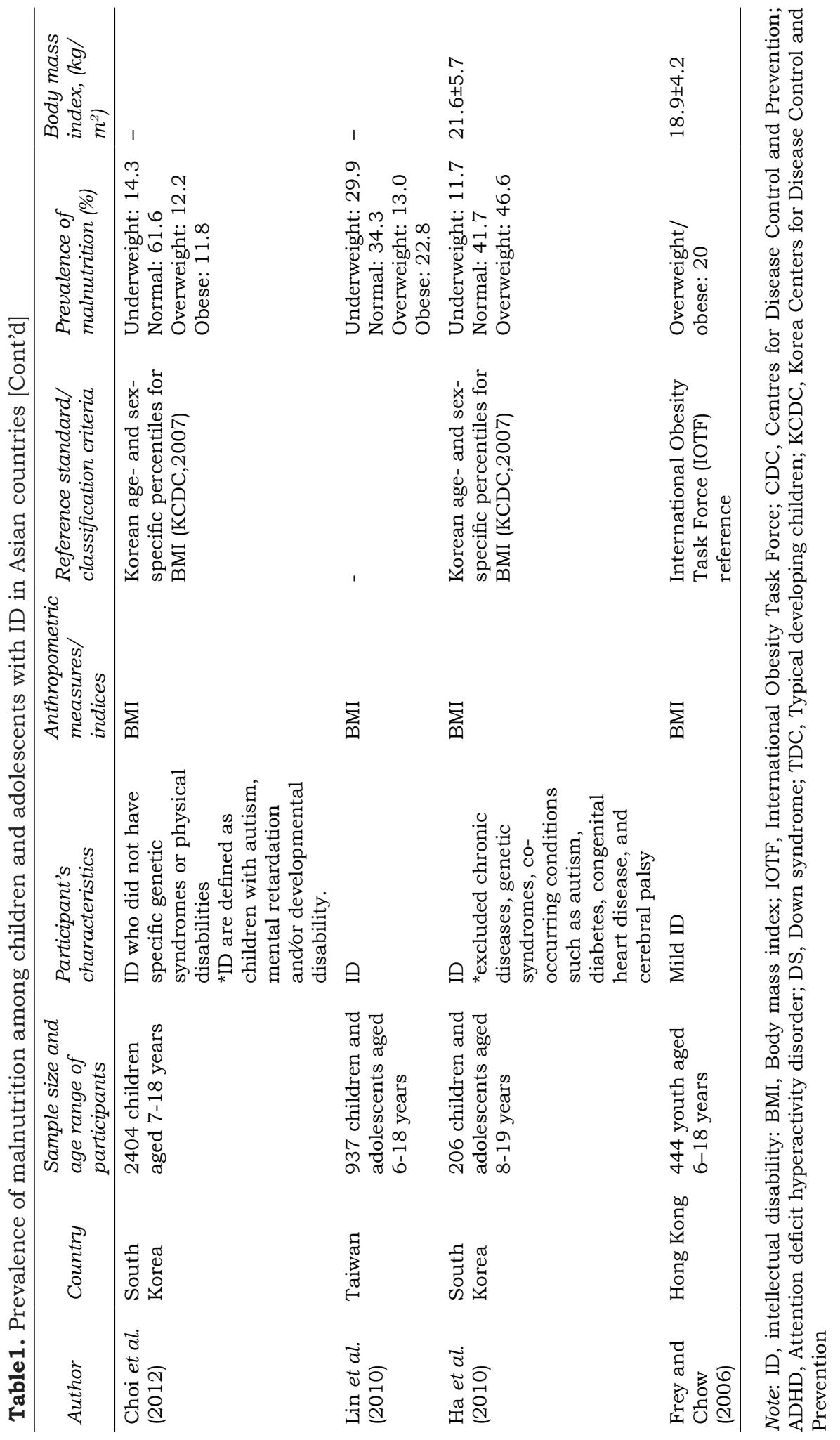


review uses the term "study" to refer to these selected articles.

\section{Geographic coverage, participants and study design}

The studies included in this review had a mixture of both samples of children and adolescents aged below 19 years, with an age range of between 1-19 years old. Four studies included samples of children under 12 years (Kavitha, Singh, \& Chandraiah, 2019; Raina et al., 2016; Sayin \& Ilik, 2017; Sulton \& Jajat, 2019), while the rest combined children and adolescents. The sample size of the participants ranged from 30-2,404 children and adolescents. Eight studies included participants with various levels of ID (mild, moderate, and severe), four studies included participants with ID and co-morbidities or other disabilities (autism, attention deficit hyperactivity disorder, epilepsy, Down Syndrome, and developmental disabilities), while five studies only mentioned participants with ID (Table 1). The level of ID was determined by using Wechsler IQ scores of below 70 (mild $=55<$ ID $<69$, moderate $=$ $40<$ ID $<54$, severe $=25<$ IQ $<39$, and profound= IQ $\leq 24)$ or obtained from school records (Kavitha et al., 2019; Pan et al., 2016; Sulton \& Jajat, 2019). The study participants were recruited from various community settings such as special education schools, households, and rehabilitation centres. These 17 studies were conducted in South Asia (Kavitha et al., 2019; Nassa \& Bhatia, 2014; Pise, Pradhan, \& Gharote, 2019; Raina et al., 2016; Sanjay \& Nadgir, 2013), Southeast Asia (Sulton \& Jajat, 2019; Tamin et al., 2014), East Asia (Choi et al., 2012; Frey \& Chow, 2006; Ha, Vann \& Choi, 2010; Lin et al., 2010; Pan et al., 2016; Wang et al., 2018), and Southwestern Asia (Nogay, 2013; Sari et al., 2016; Sari \& Bahceci, 2012; Sayin \& Ilik, 2017). No study was found for Central Asia and North Asia. The design of these selected studies was crosssectional.

\section{Measurements and reference standards}

Majority of the studies $(n=15)$ employed body mass index (BMI) to assess underweight, overweight and obesity, while only two studies used height-forage and weight-for-age to determine stunting and wasting (Nogay, 2013; Raina et al., 2016). Height and weight in majority of the studies were measured directly by the research team or obtained through school health records or measurements taken by clinicians (e.g., nurses and dietitians). These studies used a variety of reference standards, which included the International Obesity Task Force (IOTF) reference (Pan et al., 2016; Pise et al., 2019; Sayin \& Ilik, 2017; Sulton \& Jajat, 2019; Wang et al., 2018), Centers for Disease Control (CDC) 2000 growth charts (Kavitha et al., 2019; Nassa \& Bhatia, 2014; Sanjay $\&$ Nadgir, 2013) Taiwanese growth chart (Pan et al., 2016), Korea Centers for Disease Control and Prevention (KCDC) reference (Choi et al., 2012; Ha et al., 2010), Turkish BMI percentile (Sari et al., 2016; Sari \& Bahceci, 2012), and Waterlow's classification (Raina et al., 2016). Only four studies did not report the reference standards that they had used.

\section{Prevalence of malnutrition}

Based on Table 1, prevalences of underweight, overweight and obesity were reported in fifteen of the studies. Two studies published on stunting and wasting (Nogay, 2013; Raina et al., 2016), while a study reported on non-obese and obese subjects (Tamin et al., 2014). Fifteen studies examined nutritional status focusing on stunting, wasting, underweight, overweight and obesity as primary outcomes, while the rest of the studies addressed them as 
secondary outcomes (Lin et al., 2010; Sayin \& Ilik, 2017). Meanwhile, ten studies determined nutritional status/ malnutrition and its associated factors. The prevalence of malnutrition varied widely and could not be compared as these studies used different reference standards. The findings revealed that the range for overall prevalence of underweight was 5.7\%-76.3\%. Meanwhile, the prevalence estimated for overweight was $3.9 \%-46.6 \%$ and obesity was $1.6 \%-26.6 \%$. However, four studies found none of their subjects/ respondents were obese (Ha et al., 2010; Kavitha et al., 2019; Pise et al., 2019; Raina et al., 2016). Two studies reported that the prevalence of stunting was $45.0 \%$ in Indian children (Raina et al., 2016) and $28.9 \%$ among children and adolescents in Turkey (Nogay, 2013). Meanwhile, only a study by Raina et al. (2016) reported on wasting $(7.7 \%)$. Six studies reported that the value of BMI ranged from $17.4 \pm 4.3 \mathrm{~kg} / \mathrm{m}^{2}-23.0 \pm 6.3$ $\mathrm{kg} / \mathrm{m}^{2}$ (Frey \& Chow, 2006; Ha et al., 2010; Pan et al., 2016; Pise et al., 2019; Sari \& Bahceci, 2012; Sulton \& Jajat, 2019).

\section{DISCUSSION}

This scoping review identified seventeen studies published between 2006 and 2019 addressing research on malnutrition focusing on underweight, overweight and obesity among children and adolescents with ID in Asian countries (Table 1). Although substantial information were available on childhood underweight, overweight and obesity in the general population, less is known among this vulnerable group.

This review paper found that the prevalences of underweight, overweight and obesity among children and adolescents with ID varied across the studies. It was difficult to compare the prevalences as the studies used different reference standards and definitions for malnutrition. The findings suggested that the highest prevalence of undernutrition was estimated in South Asia. Most of the studies in South Asia were conducted in India and they showed a high prevalence of wasting, stunting, and underweight as compared to the other studies in different Asian countries. Sanjay \& Nadgir (2013) reported the highest prevalence of underweight at $76.3 \%$, followed by $70.5 \%$ (Pise et al., 2019), and $64.0 \%$ (Kavitha et al., 2019), while Raina et al. (2016) estimated $45.0 \%$ for stunting and $7.7 \%$ for wasting. According to the Global Hunger Index (GHI) (2019), the high rates of child undernutrition, which include rates of child stunting and wasting, were due to South Asia's higher GHI scores. The GHI Severity Scale indicated that South Asia and Africa's South of the Sahara have serious levels of hunger as both continents have the highest regional 2019 GHI scores in the world. Several studies in South Asian countries suggested that lower socio-economic status (SES), poor dietary intakes, and food insecurity become potential determinants related to undernutrition among children (Chowdhury et al., 2018; Kim et al., 2017). However, these findings could not be generalised to all countries in South Asia, as the selected studies were only conducted in India. Meanwhile, the findings in Southwest Asia estimated the lowest prevalence of underweight at 8.0\% (Sayin \& Ilik, 2017) and 5.7\% (Sari et al., 2016); yet these findings only came from one country, which was Turkey. Although most studies did not identify the socio-economic stratification, an overall greater prevalence of underweight was reported among rural and low SES children and adolescents.

Besides, the findings estimated that the highest prevalence of overweight was $46.6 \%$ in South Korea, with no 
prevalence of obesity (Ha et al., 2010). Interestingly, the researchers suggested that the prevalence of overweight among ID children in their study was almost five times higher than the prevalence of overweight among children in the general population in South Korea. Ha et al. (2010) referred to KCDC (2007) for BMI classification and the sample was only restricted to children with ID, excluding those with chronic diseases and/or genetic syndromes, co-occurring conditions such as autism, diabetes, congenital heart disease, and cerebral palsy. Meanwhile, the highest prevalence of obesity was observed in studies from Indonesia and Turkey, which were $26.6 \%$ (Sulton \& Jajat, 2019) and 26.3\% (Sari $\&$ Bahceci, 2012), respectively. However, both studies only involved a small sample size of children and adolescents with mild ID, thus did not demographically reflect the overall population in Asia. Future research may need to use larger sample sizes of children and adolescents with ID of all levels in Asia.

The prevalences of underweight, overweight and obesity were inconsistent and varied according to the classification of BMI used. We observed that the prevalence of underweight using the CDC 2000 growth chart classification ranged from $15.0 \%-76.3 \%$, which was higher than studies that used the IOTF classification (8.0\%-13.4\%), KCDC classification (11.7\%-14.3\%), and Turkish children growth chart $(5.7 \%-$ $18.4 \%)$. However, the range of overweight and obesity prevalence using the CDC 2000 growth chart classification was lower than the other classifications (1.6\%-2.7\%). The IOTF showed a slightly higher prevalence of obesity ranging from 9.2\%-26.6\%. Since many countries have their own population-specific threshold for determining BMI, a direct comparison between reference standards was invalid due to different cut-off points used in each classification. The differences between BMI classifications would affect the accuracy and precision of the tool to assess malnutrition. However, BMI is one of the most feasible methods to evaluate body composition in individuals with ID as it shows good agreement with dual-energy X-ray absorptiometry (DXA) results (Casey, 2013). Even though BMI is simple, quick and provides straightforward results, we still need to be cautious as it measures fat-free mass and body fat as one value (Nuttall, 2015).

Besides the prevalence of malnutrition, this review also observed ten studies that investigated the factors contributing to malnutrition among children and adolescents with ID in Asia. The risk factors included comorbidity factors, behavioural factors, and parental factors. Co-morbid factors such as high blood pressure, anaemia, genetic disorder, physical disability, and level of ID were found to be significantly related with malnutrition. Sari $\&$ Bahceci (2012) found the presence of high blood pressure (20.1\%) among ID adolescents in Turkey and it was associated with BMI. This finding was consistent among ID adolescents in Taiwan as they reported that the prevalence of hypertension $(11.7 \%)$ was significantly higher than the general population of the same age, and it was associated with BMI (Lin et al., 2010). Overweight and obesity with a combination of factors such as genetics, unhealthy diet and lifestyles were major risks for hypertension, diabetes and other morbidities (Jiang et al., 2016; Raghi et al., 2016). Besides that, Pan et al. (2016) reported that ID children with genetic disorders (Down syndrome) and physical disability appeared to have higher risks of being overweight and obese. Several researches suggested that Down syndrome, which has different characteristics than normal people such as short stature, thyroid problems, and 
a lower metabolic rate contributes to overweight (Bertapelli et al., 2016; Selvi et al., 2017). Meanwhile, two studies in India revealed that those who were highly intellectually disabled were malnourished (Nassa \& Bhatia, 2014; Raina et al., 2016). These co-morbid conditions may increase functional limitations, which further threatens their ability to live independently.

The other risk factor identified was behavioural factors. Wang et al. (2018) studied on behavioural factors and reported shorter sleep duration, longer sedentary activities, higher intakes of energy-dense foods, and consumption of meats, fish, and eggs were risk factors for overweight and obesity among ID children in Hong Kong. Children who spent more time on sedentary activities and being physically inactive had reduced energy expenditure as they may be doing activities such as snacking while watching television. Children with ID face many barriers to participate in physical activity due to a lack of resources, opportunities, and psychological support (Ha et al., 2010). Furthermore, higher energy intake and unhealthy eating habits call for parental attempts to help these children to modify their unhealthy lifestyles. However, this review only found one study which explored on behavioural factors. Thus, more future research needs to be conducted, especially among ID children and adolescents in Asia.

Lastly, parental factors such as parenting practices, maternal body weight, and parent's perception were found to be risk factors of overweight and obesity (Ha et al., 2010; Wang et al., 2018). For example, Wang et al. (2018) stated that parental practice on pressure to eat more was negatively associated with overweight and obesity, and this was consistent with the findings among typically developing children (Gregory,
Paxton, \& Brozovic, 2010; Wehrly et al., 2014). Parents pressuring children to eat more would cause less enjoyment of eating (Webber et al., 2010), food avoidance (Powell, Farrow, \& Meyer, 2011), negative feeding experience, and decreased consumption of certain foods which would affect a child's weight (Galloway et al., 2006). Furthermore, children may have a higher likelihood of being overweight and obese if their mothers were overweight and obese because parents would influence children's eating habits. Thus, it is helpful to assess parental factors because family provides the strongest moral support and parental involvement in the intervention will help to prevent or overcome malnutrition.

This review has some limitations. This scoping review is different from systematic review because it does not assess the quality of the selected studies. There is a possibility that the prevalence of malnutrition might be under- or overestimated due to methodological constraints. The limited number of studies meeting the inclusion and exclusion criteria and the variation of sample sizes, participants criteria, different reference standards, and geographical areas caused difficulty in generalising the findings. Besides, some information on malnutrition might be missing due to unpublished studies that were not available online.

\section{CONCLUSION}

In conclusion, this review demonstrated concern regarding the prevalence of malnutrition among children and adolescents with ID in Asian countries. The prevalence of underweight was high, especially in low SES countries such as India. Besides, the prevalence of overweight and obesity was also high in certain countries such as Indonesia and 
Korea as compared to other countries in Asia. This review also found several risk factors related to malnutrition such as co-morbidity factors, behavioural factors, and parental factors. Many potential risk factors of malnutrition such as genetic disorder, level of ID, sedentary behaviours, dietary intake, and parenting practices are understudied in this population and deserve further investigation. Early detection on the prevalence of malnutrition and its associated factors would help to address the problem thoroughly in order to prevent further rise in prevalence. Although malnutrition is an underlying health condition in some people, it can significantly impact patient's health outcomes and cost if they do not receive proper treatment. This review found evidence that children and adolescents with ID are vulnerable groups for malnutrition in Asian countries. Hence, future studies should further investigate the understudied potential risk factors of malnutrition in order to assist in developing comprehensive nutrition intervention programmes and inclusive public health systems to combat this problem in this vulnerable population.

\section{Acknowledgement}

The authors would like to thank Ms Syahidah Nazri of Universiti Sains Malaysia for her technical input in preparing this manuscript.

\section{Authors' contributions:}

SFM, involved in study conception and design, conducted data collection, analysis and interpretation, and prepared the draft of the manuscript; SKL \& DV, involved in study conception and design, critical revision and reviewed the manuscript.

\section{Conflict of interest}

The authors report no conflicts of interest in this work.

\section{References}

Bandini L, Danielson M, Esposito LE, Foley JT, Fox MH, Frey GC, Fleming RK, Krahn G, Must A, Porretta DL, Rodgers AB, Stanish H, Urv T, Vogel LC \& Humphries K (2015). Obesity in children with developmental and/or physical disabilities. Disabil Health J 8(3):309-316.

Bellamy J (2016). Lifestyle factors affecting the health of children with intellectual disability. $\mathrm{PhD}$ thesis, The University of New South Wales, Australia.

Bertapelli F, Pitetti K, Agiovlasitis S \& Guerrajunior G (2016). Overweight and obesity in children and adolescents with Down syndrome - prevalence, determinants, consequences, and interventions: A literature review. Res Dev Disabil 57:181-192.

Casey AF (2013). Measuring body composition in individuals with intellectual disability: a scoping review. $J$ Obes 1-6.

Choi E, Park H, Ha Y \& Hwang WJ (2012). Prevalence of overweight and obesity in children with intellectual disabilities in Korea. J Appl Res Intellect Disabil 25:476-483.

Chowdhury TR, Chakrabarty S, Rakib M, Saltmarsh S \& Davis KA (2018). Socio-economic risk factors for early childhood underweight in Bangladesh. Global Health 14(54):1-12.

Eisenhower AS, Baker BL \& Blacher J (2005). Preschool children with intellectual disability: Syndrome specificity, behaviour problems, and maternal wellbeing. J Intellect Disabil Res 49(Pt9):657-671.

Frey GC \& Chow B (2006). Relationship between BMI, physical fitness, and motor skills in youth with mild intellectual disabilities. Int $J$ Obes 30(5):861-867.

Galloway AT, Fiorito LM, Francis LA \& Birch LL (2006). "Finish your soup": Counterproductive effects of pressuring children to eat on intake and affect. Appetite 46(3):318-323.

Global Nutritional Report (2020). Asia Nutrition Profile. From https://globalnutritionreport. org/resources/nutrition-profiles/asia/\#profile [Retrieved June 5 2020].

Gregory JE, Paxton SJ \& Brozovic AM (2010). Pressure to eat and restriction are associated with child eating behaviours and maternal concern about child weight, but not child body mass index, in 2- to 4-year-old children. Appetite 54(3):550-556. 
Ha Y, Vann JCJ \& Choi E (2010). Prevalence of overweight and mothers' perception of weight status of their children with intellectual disabilities in South Korea. $J$ Sch Nurs 26(3):212-222.

Hume-nixon M \& Kuper H (2018). The association between malnutrition and childhood disability in low- and middle- income countries: systematic review and meta-analysis of observational studies. Trop Med Int Health 23(11):1158-1175.

International Food Policy Research Institute (2020). In: Global Hunger Index. From https:// www.globalhungerindex.org/results.html [Retrieved June 6 2020].

Jauhari P, Bhargava R, Bhave A, Kumar C \& Kumar R (2012). Comorbidities associated with intellectual disability among pediatric outpatients seen at a teaching hospital in Northern India. $J$ Policy Pract Intellect Disabil 9(1):10-16.

Jiang SZ, Lu W, Zong XF, Ruan HY \& Liu Y (2016). Obesity and hypertension. Exp Ther Med 12(4):2395-2399.

Kavitha VK, Singh KG \& Chandraiah D (2009). Nutritional assessment in developmentally retarded children of 3-10 years age group. Int $J$ Contemp Pediatrics 6(1):77-81.

Kim R, Mejía-Guevara I, Corsi DJ, Aguayo VM \& Subramanian SV (2017). Relative importance of 13 correlates of child stunting in South Asia: Insights from nationally representative data from Afghanistan, Bangladesh, India, Nepal, and Pakistan. Soc Sci Med 187:144-154.

Krause S, Ware R, Mcpherson L, Lennox N \& Callaghan MO (2016). Obesity in adolescents with intellectual disability: Prevalence and associated characteristics. Obes Res Clin Pract 10(5):520-530.

Lin JD, Lin PY, Lin LP, Hsu SW, Loh CH, Yen $\mathrm{CF}$, Fang WH, Chien WC, Tang CC \& Wu CL (2010). Prevalence and associated risk factors of anaemia in children and adolescents with intellectual disabilities. Res Dev Disabil 31:2532.

Lin PY, Lin LP \& Lin JD (2010). Hypertension, hyperglycemia, and hyperlipemia among adolescents with intellectual disabilities. Res Dev Disabil 31(2):545-550.

Maïano C, Hue O, Morin AJS \& Moullec G (2016). Prevalence of overweight and obesity among children and adolescents with intellectual disabilities: A systematic review and metaanalysis. Obes Rev 17(7):599-611.
Maulik PK, Mascarenhas MN, Mathers CD, Dua T \& Saxena S (2011). Prevalence of intellectual disability: A meta-analysis of populationbased studies. Res Dev Disabil 32(2):419-436.

McKenzie K, Milton M, Smith G \& Ouellette-Kuntz $H$ (2016). Systematic review of the prevalence and incidence of intellectual disabilities: Current trends and issues. Curr Dev Disord $\operatorname{Rep}$ 3(2):104-115.

Nassa R \& Bhatia B (2014). A comparative study on nutritional status of day scholar and hosteller adolescent boys with intellectual disability. Int J Food Sci Nutr 3(4):125-129.

Nogay NH (2013). Nutritional status in mentally disabled children and adolescents: A study from Western Turkey. Pak J Med Sci 29(2):614618.

Nuttall FQ (2015). Obesity, BMI, and health: A critical review. Nutr Today 50(3):117-128.

Ogwu SU (2012). Parentalknowledge, attitudes, and behaviors about nutrition and physical activity in relation to BMI of children with developmental disability. PhD thesis, Drexel University School of Public Health, Pennsylvania.

Pan C, Davis R, Nichols D, Hwang SH \& Hsieh K (2016). Prevalence of overweight and obesity among students with intellectual disabilities in Taiwan : A secondary analysis. Res Dev Disabil 53-54:305-313.

Pise V, Pradhan B \& Gharote M (2019). Body mass index status among children with intellectual disability. Int $J$ Physiol Nutr Phys Educ 4(1):106-108.

Powell FC, Farrow CV \& Meyer C (2011). Food avoidance in children. The influence of maternal feeding practices and behaviours. Appetite 57(3):683-692.

Raghi HAE, Abdel-aziz SB, Shalaby SF \& El-khider RK (2016). Prevalence of obesity in mentally disabled children attending special education institutes in Khartoum State. $J$ Child Obes 1(14): $1-8$.

Raina SK, Sharma S, Bhardwaj A, Singh M, Chaudhary S \& Kashyap V (2016). Malnutrition as a cause of mental retardation : A population-based study from Sub-Himalayan India. J Neurosci Rural Pract 7:341-345.

Rimmer JH, Yamaki K, Lowry BMD, Wang E \& Vogel LC (2010). Obesity and obesity-related secondary conditions in adolescents with intellectual/developmental disabilities. $J$ Intellect Disabil Res 54(9):787-794. 
Sanjay P \& Nadgir A (2013). To find the prevalence of obesity and overweight among children having mental retardation in age group 5 to 15 years in Dharwad Urban. Int $J$ Health Sci Res 3(1):7-13.

Sari HY \& Bahceci B (2012). Nutritional status of children with an intellectual disability. Int $J$ Disabil Hum Dev 11(1):17-21.

Sari HY, Yilmaz M, Serin E, Kisa SS, Yesiltepe Ö, Tokem Y \& Rowley H (2016). Obesity and hypertension in adolescents and adults with intellectual disability. Acta Paul Enferm 29(2):169-177.

Sayin K \& Ilik S (2017). Dietary patterns and feeding problems of Turkish children with intellectual disabilities and typically developing children. J Educ Pract 8(11):123-129.

Segal M, Eliasziw M, Phillips S, Bandini L, Curtin C, Kral TVE, Sherwood NE, Sikich L, Stanish $\mathrm{H}$ \& Must A (2017). Intellectual disability is associated with increased risk for obesity in a nationally representative sample of U.S. children. Disabil Health J 9(3):392-398.

Selvi PG, Srinivasan KR, Koteeswary P, Shastri D \& Babu SK (2017). Prevalence of overweight and obesity in Down Syndrome. Int $J$ Health Sci Res 7(7):75-79.

Shea SE (2012). Intellectual disability (mental retardation). Pediatr Rev 33(3):377-398.

Sulton K \& Jajat J (2019). Relationship between gross motor skills and body mass index of children with intellectual disability. Advances in Health Sciences Research 11:210-213.

Tamin TZ, Idris FH, Mansyur M \& Syarif DR (2014). Prevalence and determinants of obesity in students with intellectual disability in Jakarta. Med J Indones 23(2):106-111.

Tricco AC, Lillie E, Zarin W, O’Brien KK, Colquhoun H, Levac D, Moher D, Peters MDJ, Horsley T, Weeks L, Hempel S, Akl EA, Chang C, McGowan J, Stewart L, Hartling L, Aldcroft A, Wilson MG, Garritty C, Lewin S, Godfrey CM, MacDonald MT, Langlois EV, Soares-Weiser K, Moriarty J, Clifford T, Tunçalp Ö \& Straus SE (2018). PRISMA extension for scoping reviews (PRISMA-ScR): Checklist and explanation. Ann Intern Med 169(7):467-473.
UNICEF (2018). In: Disabilities. The United Nations Children's Fund. From https://www.unicef. org/disabilities/. [Retrieved December 26 2019].

Wang J, Gao Y, Kwok HHM, Huang WYJ, Li S \& Li L (2018). Children with intellectual disability are vulnerable to overweight and obesity: A cross-sectional study among Chinese children. Child Obes 14(5):1-11.

Webber L, Hill C, Cooke L, Carnell S \& Wardle J (2010). Associations between child weight and maternal feeding styles are mediated by maternal perceptions and concerns. Eur J Clin Nutr 64(3):259-265.

Wehrly SE, Bonilla C, Perez M \& Liew J (2014). Controlling parental feeding practices and child body composition in ethnically and economically diverse preschool children. Appetite 73:63-171.

WHO (2020). In: Malnutrition. World Health Organization. From https://www.who.int/ news-room/fact-sheets/detail/malnutrition. [Retrieved June 6 2020].

WHO (2020). In: Definition: intellectual disability. World Health Organization. From http:// www.euro.who.int/en / health-topics / noncommunicable-diseases / mental-health/ news / news / 2010 / 15/childrens-right-tofamily-life/definition-intellectual-disability. [Retrieved June 6 2020] 